Mathematical Research Letters 9, 725-739 (2002)

\title{
COMPACTIFICATIONS OF SYMMETRIC AND LOCALLY SYMMETRIC SPACES
}

\author{
ARMAnd Borel AND Lizhen Ji
}

\section{$\S 1$. Introduction}

In this Note, $G$ is a connected non-compact linear real semisimple Lie group, $K$ a maximal compact subgroup of $G$, and $\Gamma$ an arithmetically defined not cocompact subgroup of $G$ (assumed then to be defined over $\mathbb{Q}$ ). Recall that $K$ is the fixed point set of a Cartan involution, $X=G / K$ is a complete, simply connected Riemannian manifold with non-positive curvature, and any such symmetric space without euclidean factor can be obtained in this way. We will also consider more generally pseudoriemannian symmetric spaces $Y=G / H$ (4.3, $\S \S 5,6,7)$, where $H$ is the noncompact fixed point set of an involution of $G$. The noncompact locally symmetric space $\Gamma \backslash X=\Gamma \backslash G / K(\S \S 8,9)$ has finite volume with respect to an invariant metric.

We are concerned with various compactifications of the spaces $G / K, G / H$, $\Gamma \backslash G / K$ and $\Gamma \backslash G$. Our purpose here is to announce some new results or new points of view, in particular a uniform construction of most of known compactifications of both symmetric and locally symmetric spaces, to be included in a comprehensive exposition [BJ4]. An extended survey is given in [BJ3] and a number of proofs in [BJ1] or [BJ2].

By a compactification of $X$ or $Y$ is meant a compact $G$-space with finitely many orbits containing the given space as an open orbit. There are two main cases depending on whether the space is dense or not, but the compactification is smooth in the latter case. There is a similar division for the compactifications of $\Gamma \backslash X$.

A method often used to compactify $X$, to be referred to as the embedding method, is to embed $X$ equivariantly into a compact $G$-space and take the closure. In that case, it is clear that the compactification is a compact Hausdorff $G$-space and the problem is to investigate what has been added on the boundary. Examples are the Satake compactifications of X (3.1), the Furstenberg compactifications of $\mathrm{X}$ (not reviewed here), the De Concini-Procesi wonderful compactification $\bar{X}_{c}^{W}$ of $X_{c}=G_{c} / K_{c}$ (5.1) or more generally of $G_{c} / H_{c}(\S 7)$ and the embedding of $\Gamma \backslash G$, for $\Gamma$ maximal, in the space of closed subgroups of $G$ (9.5). Of particular interest is the set of real points of the wonderful compactification $(5.2,5.3)$ and its comparison with the Oshima-Sekiguchi $\bar{X}^{O S}$ compactification

Received May 21, 2002. 
(6.5). We shall also emphasize another procedure, the "attachment method", which can in fact be used to define most compactifications considered here. Roughly speaking, it consists in defining a priori some pieces of the boundary, usually (though not always) parametrized by parabolic subgroups. One has then to show that these pieces combine to define a compact Hausdorff $G$-space. Often, the Hausdorff property is the most difficult to establish. Examples are the conic (geodesic) or Martin compactifications of X (3.5), the Satake compactifications of $\Gamma \backslash X(8.2)$, the Borel-Serre and reductive Borel-Serre compactifications of $\Gamma \backslash X(8.3,8.4)$ or of $\Gamma \backslash G(9.1,9.2)$ and an alternate definition of the maximal Satake compactification of X $(3.2,3.3)$.

In these compactifications, except for $\bar{X}_{c}(\mathbb{R})$, the initial space is dense and the compactification has in general singularities. The first constructions of smooth analytic compactifications are due to Oshima [O] and Oshima-Sekiguchi [OS]. Their motivation was to supply a framework for the proof, by means of differential systems with regular singularities, of the Helgason conjecture on joint eigenfunctions of invariant differential operators for $G / K$, and of its generalizations to $G / H$. Besides their method, we shall also use the self-gluing of manifolds with corners (a procedure initiated by R. Melrose) and the investigation of real points of the De Concini and Procesi wonderful compactification. The latter gives a direct construction of the analytic structure of the Oshima and the Oshima-Sekiguchi compactifications.

\section{$\S 2$. Preliminaries}

We assume familiarity with the structure theory of semisimple real Lie groups and recall some notation. A maximal compact subgroup $K$ is fixed and $\theta$ is the Cartan involution of $G$ with respect to $K$.

2.1. The Lie algebra of a real Lie group $G, H, K, \ldots$ is denoted by the corresponding l.c. Gothic letter $\mathfrak{g}, \mathfrak{h}, \mathfrak{k}, \ldots$ Let $\mathfrak{a}$ be a Cartan subalgebra of the symmetric pair $(G, K)$, i.e. a maximal commutative subalgebra of the orthogonal complement $\mathfrak{s}$ to $\mathfrak{k}$ in $\mathfrak{g}$, (with respect to the Killing form). Let $A=\exp \mathfrak{a}$ and $\Phi=\Phi(G, A)$ be the set of roots of $G$ with respect to $A$. The value of $\alpha \in \Phi$ on $a \in A$ is denoted $a^{\alpha}$. As usual

$$
\mathfrak{g}_{\alpha}:=\left\{X \in \mathfrak{g} \mid \operatorname{Ad} a \cdot X=a^{\alpha} \cdot X\right\}
$$

(and a non-trivial character $\beta$ of $A$ is a root if and only if $\mathfrak{g}_{\beta} \neq 0$ ). If $P$ is a parabolic subgroup, we let $P=N_{P} \cdot A_{P} \cdot M_{P}$ be its Langlands decomposition : $N_{P}$ is the unipotent radical of $P, A_{P} \cdot M_{P}=\mathcal{Z}\left(A_{P}\right)$ the centralizer of $A_{P}$ in $G$ and also the Levi subgroup of $P$ stable under $\theta, A_{P}$ the exponential of the intersection of $\mathfrak{s}$ with the center of the Lie algebra of $\mathcal{Z}(A)$ and $M_{P}$ the direct complement of $A_{P}$ in $\mathcal{Z}\left(A_{P}\right)$ stable under $\theta$. Let $K_{P}=K \cap M_{P}$. It is a maximal compact subgroup of $M_{P}$ and $X_{P}=M_{P} / K_{P}$ is the symmetric space of noncompact type of $M_{P}$. Since $G=P \cdot K$, the projection $G \rightarrow X$ induces a surjective map

$$
\mu_{P}: N_{P} \times A_{P} \times X_{P} \rightarrow X
$$


which is an isomorphism of smooth manifolds.

2.2. We fix an ordering on $\Phi$ and let $\Delta$ be the set of simple roots. Let $N$ be the connected group with Lie algebra $\mathfrak{n}=\oplus_{\alpha>0} \mathfrak{g}_{\alpha}$. For any subset $J$ of $\Delta$, let $A_{J}=\left(\bigcap_{\alpha \in J} \operatorname{ker} \alpha\right)^{o}$, where ${ }^{o}$ refers to the identity component, and $P_{J}=$ $\mathcal{Z}\left(A_{J}\right) \cdot N$. The $P_{J}$ are the standard parabolic subgroups and any parabolic subgroup is conjugate to one and only one $P_{J}$. The Langlands decomposition of $P_{J}$ is denoted $P_{J}=N_{J} \cdot A_{J} \cdot N_{J}$. If $J=\emptyset$ we write simply $P_{\emptyset}=N \cdot A \cdot M$. In this case, $M$ is the biggest compact subgroup of $\mathcal{Z}(A)$.

2.3. Given a parabolic subgroup $P$, we let $\Phi\left(P, A_{P}\right)$ denote the set of weights of $A_{P}$ in $\mathfrak{n}_{P}$, with respect to the adjoint representation. They are positive integral linear combinations of a subset $\Delta\left(P, A_{P}\right)$ of cardinality $\operatorname{dim} A_{P}$, called the simple elements of $\Phi\left(P, A_{P}\right)$. [If $P=P_{J}$, these are the restrictions of the elements of $\Delta-J$.$] For t>0$, we let

$$
A_{P, t}=\left\{a \in A_{P} \mid a^{\alpha} \geq t, \alpha \in \Phi\left(P, A_{P}\right)\right\}=\left\{a \in A_{P} \mid a^{\alpha} \geq t, \alpha \in \Delta\left(P, A_{P}\right)\right\} .
$$

For bounded sets $U \subset N_{P}$ and $V \subset X_{P}$, the image of $U \times A_{P, t} \times V$ under $\mu_{P}$ is called a Siegel set in $X$, with respect to $P$, to be denoted $\mathfrak{S}_{P, U, t, V}$.

\section{$\S 3$. Satake compactifications and an alternative construction}

Compactifications of $X$ were constructed first by I. Satake [S1] (as a tool to obtain compactifications of $\Gamma \backslash X$ [S2], see 8.2 below) and later other compactifications were introduced. See [GJT] or [BJ3] for an exposition. In this section we concentrate on the compactifications of Satake.

3.1. For simplicity we assume $G$ to be simple and recall first Satake's definition. Given a finite dimensional complex vector space $V$, let $\mathcal{H}_{V}$ be the space of nonzero hermitian forms on $V$ and $P\left(\mathcal{H}_{V}\right)=\mathcal{H}_{V} / \mathbb{R}^{*}$ the associated real projective space. Let now $\rho: G \rightarrow G L(V)$ be a finite dimensional non-trivial irreducible smooth representation of $G$ in $G L(V)$. It is always possible to find a positive definite hermitian metric on $V$ such that $\rho(x)$ is unitary (resp. hermitian) if $x \in K$ (resp. $x \in S=\exp \mathfrak{s}$ ). Then the map $g \mapsto \rho(g) \cdot \rho(g)^{*}$, where ${ }^{*}$ refers to the adjoint, yields an embedding of $X$ into $P\left(\mathcal{H}_{V}\right)$. The closure of $\rho(X)$ is the compactification $\bar{X}_{\rho}^{S}$ of $X$ associated to $\rho$ [S1]. Up to isomorphism, it depends only on the set $I$ of simple roots not orthogonal to the highest weight $\lambda_{\rho}$ of $\rho$ and will also be denoted $\bar{X}_{I}^{S}$. If $I \subset I^{\prime}$, the compactification $\bar{X}_{I^{\prime}}^{S}$ dominates $\bar{X}_{I}^{S}$, in the sense that the identity map $X \rightarrow X$ extends to a continuous surjective map $\bar{X}_{I}^{S} \rightarrow \bar{X}_{I^{\prime}}^{S}$. In particular, if $I=\Delta$, then $\bar{X}_{I}^{S}$ dominates all other Satake compactifications, and is called the maximal Satake compactification, to be denoted $\bar{X}_{m}^{S}$. For $J \subset \Delta$, let $K_{J}=K \cap M_{J}$. It is maximal compact in $M_{J}$. Let $Q_{J}=N_{J} \cdot A_{J} \cdot K_{J}$. Then the $G$-orbits in $\bar{X}_{m}^{S}$ are the quotients $O_{J}=G / Q_{J}$ :

$$
\bar{X}_{m}^{S}=\coprod_{J \subset \Delta} O_{J}
$$


(If $J=\Delta$, then $P_{J}=G$ and $K_{J}=K$.) The orbit $O_{J}$ is fibered over $G / P_{J}$ with fibers isomorphic to $X_{J}=M_{J} / K_{J}$, called the "boundary components".

If $I \neq \Delta$, the compactification $\bar{X}_{I}^{S}$ has a similar description, but $J$ runs only through certain subsets of $\Delta$ and $X_{J}$ is replaced by a suitable direct factor, to be denoted here $X_{J}^{\prime}$, or $X_{P}^{\prime}$ if $P$ is not standard.

3.2. We now sketch another way to define $\bar{X}_{m}^{S}$ by the attachment method (see [BJ2] for details). Start from a set $\tilde{X}$ which is the union of $X$ and of boundary faces $e(P)$, where $e(P)$ is isomorphic to $X_{P}$. By definition, a sequence of points $y_{j} \in X$ converges to $z \in e(P)$ if the following conditions are fulfilled: $y_{j}$ can be written in the form

$$
y_{j}=k_{j} \cdot \mu_{P}\left(n_{j}, a_{j}, z_{j}\right) \quad\left(k_{j} \in K, n_{j} \in N_{P}, a_{j} \in A_{P}, z_{j} \in X_{P}\right)
$$

so that $k_{j} \rightarrow 1, a^{\alpha} \rightarrow \infty$ for all $\alpha \in \Delta\left(P, A_{P}\right), a_{j}^{-1} \cdot n_{j} \cdot a_{j} \rightarrow 1$, and $z_{j} \rightarrow z$. Moreover, if $P_{1} \subset P_{2}$, then $e\left(P_{1}\right)$ is to be contained in the closure of $e\left(P_{2}\right)$ and the definition of convergent sequences in $e\left(P_{2}\right)$ to points of $e\left(P_{1}\right)$ is similar, using relative Langlands decompositions. It is then proved that $\tilde{X}$ is a compact Hausdorff $G$-space, isomorphic to $\bar{X}_{m}^{S}$. In (2), the representation of $y_{j}$ is not unique, and, accordingly, the Hausdorff property is not obvious, but follows from the separation property in Proposition 3.3 below. Fix on $N_{P}$ a left-invariant metric $d$ and, for $\varepsilon>0$, let $B(\varepsilon)$ be the ball of radius $\varepsilon$ in $N_{P}$ with center at the identity. A generalized Siegel set $\mathfrak{S}_{P, \varepsilon, t, V}$, where $V$ is as in 2.3, is the image under $\mu_{P}$ of

$$
\left\{(n, a, z) \in N_{P} \times A_{P} \times X_{P} \mid a \in A_{P, t}, z \in V, a^{-1} n a \in B(\varepsilon)\right\} .
$$

The chief separation property is then given by the

3.3 Proposition. Fix two distinct proper parabolic subgroups $P_{1}, P_{2}$ and a neighborhood $C$ of 1 in $K$ such that $k \cdot P_{1} \cdot k^{-1} \neq P_{2}$ for $k \in C$. Then for $\varepsilon$ sufficiently small and $t$ sufficiently large

$$
k \cdot \mathfrak{S}_{P_{1}, \varepsilon, t, V_{1}} \cap \mathfrak{S}_{P_{2}, \varepsilon, t, V_{2}}=\emptyset
$$

for all $k \in C$.

As the name indicates, the generalized Siegel sets include the Siegel sets (2.3). Proposition 3.3 is a generalization for generalized Siegel sets over $\mathbf{R}$ of a separation property of Siegel sets (usually associated to parabolic $\mathbf{Q}$-subgroups) familiar in reduction theory.

3.4. It is known that all Satake compactifications are homeomorphic to closed balls [J1], but $\bar{X}_{m}^{S}$ has two further interesting structures. First, the boundary admits a natural structure of simplicial complex dual to the Tits building of parabolic subgroups [GJT]. Second, and more importantly here, it is a manifold with corners. A manifold with corners $M$, of dimension $n, \operatorname{rank} r k(M)=r$ 
is a stratified space. The boundary is the union of the strata of codimension $j, 1 \leq j \leq r$. The boundary faces are the closures of the strata. A point on a boundary face of codimension $j$ has a fundamental system of neighborhoods homeomorphic to $\mathbb{R}^{n-j} \times \mathbb{R}_{\geq 0}^{j}$, where $\mathbb{R}_{\geq 0}^{j}$ denotes the closed quadrant in $\mathbb{R}^{j}$ of points with coordinates $\geq 0$.

There are further properties we do not recall (see [BS] or [BJ3]).

Embed $A$ in $\mathbb{R}^{\Delta}$ (euclidean space with coordinates labeled by $\Delta$ ) by mapping $a$ to the points with coordinates $a^{-\alpha}(\alpha \in \Delta)$. Let $\bar{A}$ be its closure. It is the closed quadrant $\mathbb{R}_{\geq 0}^{\Delta}$. Then it can be seen that $\bar{X}_{m}^{S}$ has charts of the form $N^{-} \times \bar{A}$, where $N^{-}$is the connected group with Lie algebra $\mathfrak{n}^{-}=\oplus_{\alpha<0} \mathfrak{g}_{\alpha}$. We shall see below $(4.2,5.5)$ that it admits a structure of analytic $G$-space.

3.5. Other examples of the attachment method are given in 8.2, 8.3 and 8.4. As is already indicated in [BJ2] and will be shown in [BJ4], most other compactifications described in [GJT] (conic, Martin, Karpelevic) can be handled in this way. Basically, the procedure is the same as in 3.2, but the choices of the boundary faces $e(P)$ will vary. For instance $e(P)$ is equal to $\mathfrak{a}_{P}^{+}(\infty)\left(\operatorname{resp} . \overline{\mathfrak{a}_{P}^{+}(\infty)} \times X_{P}\right)$ for the conic (resp. Martin) compactification, where $\mathfrak{a}_{P}^{+}(\infty)$ may be identified with the intersection of a positive chamber with the unit sphere in $\mathfrak{a}_{P}$. This is in particular convenient to see how they are related to one another, e.g. to show that the Martin compactification is the least common refinement of the maximal Satake compactification and of the conic compactification, and to see how compactifications of locally symmetric spaces are related to compactifications of symmetric spaces when only boundary faces $e(P)$ of rational parabolic subgroups $P$ are used.

\section{$\S 4$. The Oshima compactification of $X$ and the self-gluing of a manifold with corners}

4.1 A partition of euclidean space $([\mathrm{O}],[\mathrm{OS}]$, also reproduced in [BJ3]). As before, we consider the euclidean space $\mathbb{R}^{\Delta}$ with coordinates labeled by $\Delta$, and let $\left\{t^{\alpha}\right\}$ be the coordinates of $t \in \mathbb{R}^{\Delta}$.

The signature $\operatorname{sgn}(t)$ of $t \in \mathbb{R}$ is by definition equal to 0 if $t=0$, to $t /|t|$ otherwise. Thus, it belongs to $\{ \pm 1,0\}$. A signature $\varepsilon$ on $\Delta$ is a map of $\Delta$ into $\{ \pm 1,0\}$. Its support $s(\varepsilon)$ is the set of $\alpha$ on which it is not zero. It is proper if $s(\varepsilon)=\Delta$. We let $\mathcal{E}(\Delta)$ and $\mathcal{E}^{o}(\Delta)$ be respectively the set of signatures and proper signatures on $\Delta$. For $J \subset \Delta$, an element of $\mathcal{E}(J)$ will be identified with the signature of $\Delta$ with support contained in $J$, which is equal to $\varepsilon$ on $J$. For $t \in \mathbb{R}^{\Delta}$, the signature $\varepsilon_{t}$ is defined by $\varepsilon_{t}(\alpha)=\operatorname{sgn}\left(t^{\alpha}\right)$. We also write $s(t)$ for $s\left(\varepsilon_{t}\right)$. If $\varepsilon \in \mathcal{E}(\Delta)$, let

$$
\mathbb{R}^{\Delta, \varepsilon}=\left\{t \in \mathbb{R}^{\Delta} \mid \operatorname{sgn}(t)=\varepsilon\right\}
$$

It is an open quadrant in $\mathbb{R}^{J}(J=s(\varepsilon))$ and the $\mathbb{R}^{\Delta, \varepsilon}$ form a partition of $\mathbb{R}^{\Delta}$ into $3^{r}$ subsets. 
4.2. Oshima has constructed a compactification of $X$, to be denoted here $\bar{X}^{O}$, which is a compact smooth analytic $G$-space. The open orbits are $2^{r}$ copies of $X$. The union of the non-open orbits consists of $r$ smooth closed hypersurfaces with transversal intersections (the real analogue of a divisor with normal crossings). Altogether, $\bar{X}^{O}$ is the union of $3^{r}$ orbits, of which only one, isomorphic to $G / P_{\phi}$, is closed. We recall briefly its definition. First define a map $a: \mathbb{R}^{\Delta} \rightarrow A$ by the rule

$$
a(t)=\exp -\sum_{\alpha \in s(t)}\left|\ln \left(t^{\alpha}\right)\right| \alpha^{\vee}
$$

(where $\alpha^{\vee}$ is the coroot corresponding to $\alpha$ ). Then, by definition $\bar{X}^{O}$ is the quotient of $G \times \mathbb{R}^{\Delta}$ by the equivalence relation

$$
(g, t) \sim\left(g^{\prime}, t^{\prime}\right) \Longleftrightarrow(i) \varepsilon_{t}=\varepsilon_{t^{\prime}} \text { and }(i i) g \cdot a(t) \cdot Q_{s(t)}=g^{\prime} \cdot a\left(t^{\prime}\right) \cdot Q_{s\left(t^{\prime}\right)} \text { in } G \text {. }
$$

(See 3.1 for $Q_{s(t)}$. Note that $s(t) \subset \Delta$, and that $s(t)=s\left(t^{\prime}\right)$ by $(i)$.)

The $G$-action is defined by left-translations on the first factor. It is easily seen that the closure of an open orbit is isomorphic to $\bar{X}_{m}^{S}$.

4.3. The space $\bar{X}_{m}^{S}$ is a manifold with corners (3.4) and the corner structure is in a way the same as that of the positive closed quadrant $\mathbb{R}^{\Delta}$. This allows one to give a direct construction of $\bar{X}^{O}$. We sketch it.

Given two signatures $\varepsilon, \varepsilon^{\prime}$, we write $\varepsilon \subset \varepsilon^{\prime}$ if $s(\varepsilon) \subset s\left(\varepsilon^{\prime}\right)$ and $\varepsilon^{\prime}$ coincide with $\varepsilon$ on $s(\varepsilon)$. For $\delta \in \mathcal{E}^{o}(\Delta)$, let $\mathcal{E}_{\delta}(\Delta)$ be the set of $\varepsilon$ such that $\varepsilon \subset \delta$. Clearly, such an $\varepsilon$ is completely characterized by its support, which is arbitrary, therefore

$(*)$ the map $\varepsilon \mapsto s(\varepsilon)$ defines a bijection between $\mathcal{E}_{\delta}(\Delta)$ and the set of subsets of $\Delta$.

Let now $\tilde{X}$ be the disjoint union of spaces $O_{J, \varepsilon}$, where $\varepsilon$ has support $J(J \subset \Delta)$. For $\delta \in \mathcal{E}^{o}(\Delta)$, let $\tilde{X}(\delta)$ be the union of the $O_{J, \varepsilon}(\varepsilon \subset \delta)$. The remark $(*)$ above shows the existence of a canonical bijection of $\tilde{X}(\delta)$ onto $\bar{X}_{m}^{S}$. We use it to endow $\tilde{X}(\delta)$ with the topology of $\bar{X}_{m}^{S}$. Let $\delta, \delta^{\prime} \in \mathcal{E}^{o}(\Delta)$. The intersection $\tilde{X}(\delta) \cap \tilde{X}\left(\delta^{\prime}\right)$ is the union of the $O_{J, \varepsilon}$ where $\varepsilon$ runs through $\mathcal{E}_{\delta}(\Delta) \cap \mathcal{E}_{\delta^{\prime}}(\Delta)$. The topologies induced by those of $\tilde{X}(\delta)$ and $\tilde{X}\left(\delta^{\prime}\right)$ on their intersection are therefore the same. We then endow $\tilde{X}$ with the sum of the topologies of the $\tilde{X}(\delta)$. It is easily seen that a neighborhood of $x \in O_{J, \varepsilon}$ is the union of the $\tilde{X}(\delta)(\delta \supset \varepsilon)$ and that $x$ is a smooth point on it.

4.4. Let $M$ be a manifold with corners of rank $r$, as in 3.1. Call boundary hypersurfaces the closures of the strata of codimension one. Assume they are embedded (no self-intersection) and that the set $\mathcal{H}_{M}$ of boundary hypersurfaces has a finite partition

$$
\mathcal{H}_{M}=\coprod_{1 \leq j \leq N} \mathcal{H}_{j}
$$


where the elements of $\mathcal{H}_{j}$ are disjoint. Such a partition obviously exists if $M$ is compact. If not, it is assumed. Of course $N \geq r$. R. Melrose has given an inductive procedure to glue a certain number $q\left(2^{r} \leq q \leq 2^{N}\right)$ of copies of $M$ along the $\mathcal{H}_{j}$ to get a manifold (cf. [BJ1], [BJ3]). We are concerned here only with cases where $r=N$. Then $2^{r}$ copies are glued by a procedure similar to the one used above, in which the strata play the role of the $O_{J}$. In the case of $\bar{X}_{m}^{S}$, each $\mathcal{H}_{j}$ consists of one element, the closure of $O_{\Delta-\{\alpha\}},(\alpha \in \Delta)$.

\section{$\S 5$. The wonderful compactification of $G_{c} / K_{c}$ and its real points}

5.1. Given a connected complex semisimple group $Q$ and the fixed point set $R$ of an involution of $Q, \mathrm{C}$. De Concini and C. Procesi have constructed a so-called wonderful compactification of $Q / R$, to be denoted $(\overline{Q / R})^{W}$, which is a smooth projective variety $[\mathrm{CP} 1],[\mathrm{CP} 2]$.

Two definitions are given in [CP1]. One is the closure of the orbit of $\mathfrak{r}$ in the Grassmannian of $\operatorname{dim} \mathfrak{r}$ subspaces of $\mathfrak{q}$, acted upon via the adjoint representation. Another one is a definition in terms of linear representations which may be viewed as a complex analogue of Satake's procedure.

In this section, we concentrate on the case where $Q=G_{c}$ and $R=K_{c}$ are the complexifications of $G$ and $K$ and let $X_{c}=G_{c} / K_{c}$. It follows from [CP1] that $\bar{X}_{c}^{W}$ is a smooth projective irreducible variety, in which $X_{c}$ is Zariski open, with complement the union of $r$ divisors with normal crossings. Its orbit structure is a complexification of the one of $\bar{X}_{m}^{S}$ :

$$
\bar{X}_{c}^{W}=\coprod_{J \subset \Delta} O_{J, c}, \quad \text { where } O_{J, c}=G_{c} / Q_{J, c},
$$

the complex codimension of $O_{J, c}$ being Card $(\Delta-J)$. The groups $G_{c}$ and $K_{c}$ are defined over $\mathbb{R}$, hence so is $\bar{X}_{c}^{W}$. It follows from $[\mathrm{W}]$ that $\bar{X}_{c}^{W}(\mathbb{R})$ is a smooth real projective variety, on which $G$ operates with finitely many orbits. The subgroups $Q_{J, c}$ are also defined over $\mathbb{R}$ and so are the $O_{J, c}$. We have

$$
\bar{X}_{c}^{W}(\mathbb{R})=\coprod_{J \subset \Delta} O_{J, c}(\mathbb{R}) .
$$

Our goal is to describe the right hand side. To this effect, we need some preparation.

5.2 The real forms $K_{\varepsilon}$ of $K_{c}$ (cf. [OS1]). An extended signature $\tilde{\varepsilon}$ is a signature on $\Phi=\Phi(G, A)$ such that

$$
\tilde{\varepsilon}(\alpha)=\tilde{\varepsilon}(-\alpha) \quad \tilde{\varepsilon}(\alpha+\beta)=\tilde{\varepsilon}(\alpha) \tilde{\varepsilon}(\beta) .
$$

It is therefore completely determined by its restriction to $\Delta$ and any signature $\varepsilon$ on $\Delta$ extends uniquely to one $\tilde{\varepsilon}$ on $\Phi$. It is proper if and only $\varepsilon$ is proper. Given a proper signature $\varepsilon$, define a linear bijective map $\theta_{\varepsilon}$ of $\mathfrak{g}$ onto itself by the rule

$$
\theta_{\varepsilon}=\theta \text { on } \mathfrak{m} \oplus \mathfrak{a}, \quad \theta_{\varepsilon}(x)=\varepsilon(\beta) \cdot \theta(x) \quad\left(x \in \mathfrak{g}_{\beta}, \beta \in \Phi\right) .
$$


It is easily checked to be an involutive automorphism of $\mathfrak{g}$. Let then

$$
K_{\varepsilon}=G^{\theta_{\varepsilon}} .
$$

It can be shown to be a real form of $K_{c}$.

By construction $K_{\varepsilon}$ contains $M$ and is stable under $\theta$, hence the restriction of $\theta$ to $K_{\varepsilon}$ is a Cartan involution and $\mathfrak{a}$ is a Cartan subalgebra of the pseudoriemannian symmetric pair $\left(G, K_{\varepsilon}\right)$. Since $K_{\varepsilon}$ contains $M$, conjugation of $K_{\varepsilon}$ under $\mathcal{N}(A)$ amounts to conjugation under $W$ and it makes sense to speak of ${ }^{w} K_{\varepsilon}$. On the other hand, $W$ operates on $\mathcal{E}(\Phi)$ by the rule

$$
w(\varepsilon)(\beta)=\varepsilon\left(w^{-1} \cdot \beta\right) \quad(\beta \in \Phi) .
$$

Clearly, ${ }^{w} K_{\varepsilon}=K_{w(\varepsilon)}$. Using Galois cohomology, one can show

$$
\left(G_{c} / K_{c}\right)(\mathbb{R})=\coprod_{\varepsilon \in \mathcal{E}^{o}(\Phi) / W} G / K_{\varepsilon}
$$

This can be repeated for the symmetric pair $\left(M_{J}, K_{J}\right)$ with Cartan subgroup $A^{J}$ equal to the exponential of $\mathfrak{m}_{J} \cap \mathfrak{a}$, whose Weyl group $W_{J}$ is generated by the reflections $s_{\alpha}(\alpha \in J)$. One gets

$$
\left(M_{J, c} / K_{J, c}\right)(\mathbb{R})=\coprod_{\varepsilon \in \mathcal{E}^{o}(J) / W_{J}} M_{J} / K_{J, \varepsilon} .
$$

However, from the fact that $G \rightarrow\left(G_{c} / P_{J, c}\right)(\mathbb{R})$ is surjective, one deduces a natural identification between the $G$-orbits in $O_{J, c}(\mathbb{R})$ and the $M_{J}$-orbits in $\left(M_{J, c} / K_{J, c}\right)(\mathbb{R})$ so that $(2),(7),(8)$ provide a description of $\bar{X}_{c}^{W}(\mathbb{R})$.

5.3 Remark. The group $K_{\varepsilon}$ introduced in (5) is not exactly the one so denoted in [OS1], which is in general a subgroup of finite index of the present $K_{\varepsilon}$ (see 6.1). The group $K_{\varepsilon}$ is generated by the identity component $K_{\varepsilon}^{o}$ of $K_{\varepsilon}$ and the subgroup $U_{\varepsilon}$ of elements in $W$ leaving $\varepsilon$ stable, or, more correctly, by $K_{\varepsilon}^{o}$ and the inverse image of $U_{\varepsilon}$ in $\mathcal{N}(A)$. Similarly, $K_{J, \varepsilon}$, where $\varepsilon$ has support $J$ is generated by its identity component and the stability group $U_{J, \varepsilon}$ of $\varepsilon$ in $W_{J}$.

5.4 Example. Let $G=\mathbf{S L}_{n}(\mathbb{R}), K=\mathbf{S O}_{n}$. Then the $K_{\varepsilon}$ are the special orthogonal groups of non-degenerate quadratic forms on $\mathbb{R}^{n}$ of arbitrary index (or sometimes finite extensions of them). The space $\bar{X}_{c}^{W}$ is called in [CGMP] the space of complete quadrics. A point represents a flag in $\mathbb{C}^{n}$ endowed with nondegenerate quadrics on the successive quotients. Similarly, the points in $\bar{X}_{c}^{W}(\mathbb{R})$ represent flags in $\mathbb{R}^{n}$ endowed with non-degenerate real quadrics (or quadratic forms up to a factor in $\mathbb{R}^{*}$ ) on the successive quotients.

5.5. The action of $G$ on $\bar{X}_{c}^{W}(\mathbb{R})$ is algebraic, in particular analytic. On the other hand, one sees from 3.1(1) and (7), (8) above that the closure of $X$ in $\bar{X}_{c}^{W}(\mathbb{R})$ (identified to the orbit of the origin) is $\bar{X}_{m}^{S}$, whence a structure of real analytic $G$-space on the latter. These analytic structures on the pieces $\tilde{X}(\delta)$ in 4.3 combine to define an analytic $G$-space structure on $\tilde{X}$. This provides an alternate proof of Oshima's theorem in [O]. 
5.6 Remark. Some of the spaces $\bar{X}_{c}^{W}(\mathbb{R})$ also occur in [EL], from a completely different point of view.

\section{$\S 6$. The Oshima-Sekiguchi compactification and comparison with $\bar{X}_{c}^{W}(\mathbb{R})$}

6.1. Let $\varepsilon \in \mathcal{E}(\Delta)$ and $\tilde{\varepsilon} \in \mathcal{E}(\Phi)$ its extension to $\Phi$ (5.2). It can be shown that $s(\tilde{\varepsilon})$ consists of the roots of $M_{J}$ with respect to $A^{J}$ where $J=J_{\varepsilon}$ is the support of $\varepsilon$. [OS1] introduces further a subgroup

$$
W_{\varepsilon}=\left\langle s_{\alpha}, \varepsilon(\alpha)=1, \alpha \in \Phi_{J}\right\rangle \quad\left(\Phi_{J}:=\Phi\left(M_{J}, A^{J}\right)\right)
$$

of $W_{J}$ and a canonical set of representatives $W_{J}(\varepsilon)$ of $W_{J} / W_{\varepsilon}([\mathrm{OS} 1], 2.4)$. The group $K_{J, \varepsilon}$ they define, to be denoted here $K_{J, \varepsilon}^{*}$, is generated by $K_{J, \varepsilon}^{o}$ and the subgroup $W_{J, \varepsilon}$ of $W_{J}$ defined by (1). We also set

$$
Q_{J, \varepsilon}^{*}=K_{J, \varepsilon}^{*} \cdot A_{J} \cdot N_{J} \text { and } O_{J, \varepsilon}^{*}=G / Q_{J, \varepsilon}^{*} .
$$

6.2. We assume again that $G$ is simple over $\mathbb{R}$. Then $\Phi$ is irreducible. If it is not reduced, it is of type $\mathbf{B C}_{r}$. [OS1] denotes by $W^{\prime}$ the subgroup of $W$ generated by the reflections to the roots in $\Phi$ which are neither properly multipliable or divisible in $\Phi$. It is of type $\mathbf{D}_{r}$. If $\Phi$ is reduced, let $W^{\prime}=W$. It is shown in [OS1], see 2.5(iii) that $W(\varepsilon) \subset W^{\prime}$, hence $W(\varepsilon)$ is also a set of representatives of $W_{J}^{\prime} / W_{\varepsilon}^{\prime}$, where ' refers to intersection with $W^{\prime}$.

6.3. The compactification of $X$ introduced in [OS1], to be denoted here $\bar{X}^{O S}$, is by definition the quotient of $M=G \times \mathbb{R}^{\Delta} \times W^{\prime}$ by an equivalence relation $\sim$, where $(g, t, w)$ is equivalent to $\left(g^{\prime}, t^{\prime}, w^{\prime}\right)$ if and only if

(i) $w\left(\varepsilon_{t}\right)=w^{\prime}\left(\varepsilon_{t^{\prime}}\right),(i i) w^{-1} \cdot w^{\prime} \in W(\varepsilon),($ iii $) g \cdot a(t) \cdot Q_{t}^{*} \cdot w^{-1}=g^{\prime} \cdot a\left(t^{\prime}\right) \cdot Q_{t^{\prime}}^{*} \cdot w^{\prime-1}$,

the $G$-action being defined by left translations on the first factor.

[We have written $Q_{t}^{*}$ for $Q_{J_{t}, \varepsilon_{t}}^{*}$. Note that (i) implies ${ }^{w} Q_{t}^{*}={ }^{w^{\prime}} Q_{t^{\prime}}^{*}$, so that (iii) makes sense in $G$.]

6.4. By the main theorem 2.7 of $[\mathrm{OS} 1], \bar{X}^{O S}$ is a compact Hausdorff analytic $G$-space. The $G$-orbits are isomorphic to the $O_{J, \varepsilon}^{*}$. There are $\left|W^{\prime}\right|$ closed orbits isomorphic to $G / P_{\phi}$, and $\left|W^{\prime}\right|$ open orbits isomorphic to $X$. The number of orbits isomorphic to $O_{J, \varepsilon}^{*}\left(\varepsilon \in \mathcal{E}^{o}(J)\right)$ is equal to $|W|\left|W_{\varepsilon}\right|^{-1}$.

Define an action $\circ u$ of $u \in W^{\prime}$ on $G \times \mathbb{R}^{\Delta} \times W^{\prime}$ by translation on the last factor:

$$
(g, t, w) \circ u=\left(g, t, u^{-1} \cdot w\right)
$$

This provides a right action of $W^{\prime}$ which is compatible with $\sim$, as is easily seen. It therefore defines a right action of $W^{\prime}$ on $\bar{X}^{O S}$, which obviously commutes with $G$. 
6.5 Theorem. The action just defined of $W^{\prime}$ on $\bar{X}^{O S}$ is free, and the quotient $\bar{X}^{O S} / W^{\prime}$ is naturally isomorphic to $\bar{X}_{c}^{W}(\mathbb{R})$.

We only sketch some steps of the proof. It is first deduced from the definition of $\sim$ that the given action of $W^{\prime}$ on $\bar{X}^{O S}$ is free. The group $W_{J, \varepsilon}$ (see 6.1) is normal in the subgroup $U_{J, \varepsilon}$ defined in 5.3, so that the quotient $U_{j, \varepsilon} / W_{J, \varepsilon}$ acts freely via right translations on $O_{J, \varepsilon}^{*}$, with quotient $O_{J, \varepsilon}$. From that remark and $5.2(7),(8)$ follows the existence of a bijection between the orbits of $G$ on $\bar{X}^{O S} / W^{\prime}$ and on $\bar{X}_{c}^{W}(\mathbb{R})$, which is an isomorphism on each orbit. But we need a natural equivariant isomorphism.

Let $M_{1}$ be the subspace $G \times \mathbb{R}^{\Delta} \times\{1\}$ of $M$ (see 6.3 ). It is stable under the equivalence relation $\sim$. The restriction to $M_{1}$ of $\sim$ is defined by (i) and (iii) and is completely analogous to Oshima's definition (4.2). The quotient $\tilde{X}=M_{1} / \sim$ is an open subset of $\bar{X}^{O S}$ which consists of $3^{r}$ orbits, but it is not compact, (which is why $W^{\prime}$ had to be used). On the other hand, $\bar{X}_{c}^{W}$ can also be defined similarly as a quotient of $G_{c} \times \mathbb{C}^{n}$ by an equivalence relation similar to $\sim$ ([Sp], $\S 4)$. It is then possible to define a map of $G \times \mathbb{R}^{\Delta}$ onto $G \times \mathbb{R}^{r}$ which is compatible with the two equivalence relations and yields the desired isomorphism.

\section{$\S 7$. Compactification of $G / H$}

7.1. In this section, $H$ is the fixed point set of an involution $\sigma \neq 1$ of $G$ (it could also be of finite index in such a subgroup). We assume $K$ chosen so that $\theta$ commutes with $\sigma$, which is always possible. We have then the orthogonal decompositions

$$
\mathfrak{g}=\mathfrak{k} \oplus \mathfrak{s}=\mathfrak{h} \oplus \mathfrak{q},
$$

where $\mathfrak{s}$ and $\mathfrak{q}$ are the $(-1)$-eigenspaces of $\theta$ and $\sigma$ respectively. There exists a Cartan subalgebra $\mathfrak{a}_{\iota}$ of $\mathfrak{g}$ such that

$$
\mathfrak{a}_{\theta}=\mathfrak{a}_{\iota} \cap \mathfrak{s}, \mathfrak{a}_{\sigma}=\mathfrak{a}_{\iota} \cap \mathfrak{q} \quad \mathfrak{a}_{\theta, \sigma}=\mathfrak{a}_{\iota} \cap \mathfrak{s} \cap \mathfrak{q}
$$

are maximal abelian subalgebras in $\mathfrak{s}, \mathfrak{q}$ and $\mathfrak{s} \cap \mathfrak{q}$. Any two such are conjugate under $K \cap H$. To avoid repetitions, let us introduce the set

$$
\Sigma=\{\iota, \theta, \sigma,(\theta, \sigma)\}
$$

For each $\Lambda \in \Sigma$, we have a root system $\Phi_{\Lambda}=\Phi_{\Lambda}\left(\mathfrak{g}, \mathfrak{a}_{\Lambda}\right)$. In particular $\Phi_{\iota}$ is the absolute root system, $\Phi_{\theta}$ the root system of the pair $(G, K)$. We choose compatible orderings so that simple roots map to simple roots (or zero) under the obvious restriction maps, and let $\Delta_{\Lambda}$ be the corresponding sets of simple roots. The standard $\Lambda$-relevant parabolic subgroups of $G_{c}$ are defined in terms of subsets of $\Delta_{\Lambda}$, as in the case of $\theta$ (see 2.2) and the conjugates of the standard $\Lambda$ relevant parabolic subgroups are the $\Lambda$-relevant parabolic subgroups of $G_{c}$. The $\iota$ relevant parabolic subgroups are just all parabolic subgroups, the standard $\theta-$ relevant parabolic subgroups are all the standard parabolic subgroups which are 
defined over $\mathbb{R}$. For details on these various root systems and their relationships see [OS1], [OS2], [Sp1], [Sp2] and references given there. The notation $\Sigma, \Lambda$ is introduced in [BJ3], §14.

Real forms $H_{\varepsilon}$ of $H_{c}\left(\varepsilon \in \mathcal{E}^{o}\left(\Delta_{(\theta, \sigma)}\right)\right.$ are defined in the same way as the $K_{\varepsilon}$ (see 5.2), with $\mathfrak{a}$ replaced by $\mathfrak{a}_{\theta, \sigma}$ and $\theta$ by $\sigma$ ([BJ3], 11.2).

7.2. Let $Y=G / H$. The wonderful compactification $\bar{Y}_{c}^{W}$ is described as in 5.1 in terms of standard $\sigma$-relevant parabolic subgroups. It is a smooth projective variety in which $Y_{c}$ is open and its complement is the union of $r_{\sigma}=\operatorname{dim} \mathfrak{a}_{\sigma}$ smooth divisors with normal crossings.

The projective variety $\bar{Y}_{c}^{W}$ is defined over $\mathbb{R}$ and we can consider its set of real points $\bar{Y}_{c}^{W}(\mathbb{R})$. It follows from 1.8 in [OS1] that if an orbit of $G_{c}$ contains real points, then the underlying standard parabolic subgroup is also $\theta$-relevant, so that these orbits are parametrized by the subsets of $\Delta_{(\theta, \sigma)}$ and the corners in $\bar{Y}_{c}^{W}(\mathbb{R})$ have rank equal to the dimension of $\mathfrak{a}_{\theta, \sigma}$. This yields in particular an analytic structure of $G$-space on the closure of $Y$, which is a manifold with corners. The space $Y_{c}(\mathbb{R})$ is again a union of orbits $G / H_{\varepsilon}$, and similarly for the non-open orbits in $\bar{Y}_{c}^{W}(\mathbb{R})$. A precise parameterization analogous to 5.2(7), (8) in the case $\theta=\sigma$ is likely, but has not yet been established. Also to be investigated is its relation to the closure of $Y$ in a compactification of $G / H$ defined and studied by T. Oshima [O2], a generalization of $\bar{X}^{O}$ in which all open orbits are isomorphic to $G / H$.

\section{$\S$ 8. Compactifications of $\Gamma \backslash X$}

We now assume that $G=\mathbf{G}(\mathbb{R})^{\circ}$, where $\mathbf{G}$ is a linear algebraic group defined over the field $\mathbb{Q}$ of rational numbers and $\Gamma \subset \mathbf{G}(\mathbb{Q})$ is a non-cocompact arithmetic subgroup of $\mathbf{G}$. We review briefly various known compactifications of $\Gamma \backslash X$, all obtained by the attachment method.

8.1. A $\mathbb{Q}$-group will be denoted by a bold face capital letter and its group of real points by the corresponding ordinary capital letter.

Let $\mathbf{P}$ be a parabolic $\mathbb{Q}$-subgroup of $\mathbf{G}, \mathbf{N}_{\mathbf{P}}$ its unipotent radical, $\mathbf{L}_{\mathbf{P}}=\mathbf{P} / \mathbf{N}_{\mathbf{P}}$ and $\pi_{\mathbf{P}}: \mathbf{P} \rightarrow \mathbf{L}_{\mathbf{P}}$ the natural projection. It maps any Levi subgroup of $\mathbf{P}$ isomorphically onto $\mathbf{L}_{\mathbf{P}}$. We let

$$
P=N_{\mathbf{P}} \cdot M_{\mathbf{P}} \cdot A_{\mathbf{P}}
$$

be the Langlands decomposition of $P$ relative to $\mathbb{Q}$. Here, $A_{\mathbf{P}}$ is the unique $\theta$-stable lift of $\mathbf{S}(\mathbb{R})^{o}$, where $\mathbf{S}$ is a maximal central $\mathbb{Q}$-split torus of $\mathbf{L}_{\mathbf{P}}$ and $M_{\mathbf{P}}$ the unique $\theta$-stable lift of $\mathbf{M}_{\mathbf{P}}(\mathbb{R})$, where $\mathbf{M}_{\mathbf{P}}$ is the intersection of the kernels of the squares of the rational $\mathbb{Q}$-characters of $\mathbf{L}_{\mathbf{P}}$. Thus $M_{\mathbf{P}} \cdot A_{\mathbf{P}}$ is the unique $\theta$-stable Levi subgroup of $P$, and is the centralizer of $A_{\mathbf{P}}$. The subgroup $M_{\mathbf{P}} \cdot N_{\mathbf{P}}$ of $P$ contains all arithmetic or compact subgroups of $P$. This decomposition is closely related to the Langlands decomposition $P=N_{P} \cdot M_{P} \cdot A_{P}$ of $P$ viewed as a real group (2.1). In fact, $N_{\mathbf{P}}=N_{P}$, the group $A_{\mathbf{P}}$ is contained in $A_{P}$ and 
$\pi_{P}\left(A_{P} / A_{\mathbf{P}}\right)$ is the identity component of the group of real points of the biggest central $\mathbb{Q}$-anisotropic $\mathbb{Q}$-torus of $\mathbf{L}_{\mathbf{P}}$. Accordingly, $M_{\mathbf{P}}$ is the product of $M_{P}$ by a subgroup isomorphic to $A_{P} / A_{\mathbf{P}}$.

Let $X_{\mathbf{P}}=M_{\mathbf{P}} /\left(K \cap M_{\mathbf{P}}\right)$. In analogy with $2.1(2)$, the projection $G \rightarrow X$ induces an isomorphism of smooth manifolds

$$
\mu_{\mathbf{P}}: N_{P} \times X_{\mathbf{P}} \times A_{\mathbf{P}} \stackrel{\sim}{\rightarrow} X
$$

It is of course closely related to $2.1(2)$. In fact $X_{\mathbf{P}}$ is the product of $X_{P}$ by a flat space isomorphic to $A_{P} / A_{\mathbf{P}}$.

8.2. To define a compactification of $\Gamma \backslash X$, [S2] starts from a compactification $\bar{X}_{\rho}^{S}(3.1)$ and distinguishes certain boundary components $X_{P}^{\prime}$ (see 3.1) called rational. It then considers the union $X^{*}$ of $X$ and of the rational boundary components. It is endowed with a certain topology, $\Gamma$ operates continuously on $X^{*}$ and $\Gamma \backslash X^{*}$ is the sought for compactification. It is the union of $\Gamma \backslash X$ and finitely many quotients $\Gamma_{P} \backslash X_{P}^{\prime}$, where $\Gamma_{P}$ is an arithmetically defined group of automorphisms of $X_{P}^{\prime}$. The boundary component $X_{P}^{\prime}$ is rational if first of all its normalizer $P$ is defined over $\mathbb{Q}$, and second if the centralizer of $X_{P}^{\prime}$ has a normal cocompact $\mathbb{Q}$-subgroup. In most cases in which this construction has been carried out, the second condition follows from the first. This is in particular so if $\bar{X}_{\rho}^{S}$ is the maximal Satake compactification. Then $X_{P}^{\prime}=X_{P}$. It also holds if $\bar{X}_{\rho}^{S}$ is the Baily-Borel-Satake compactification of an irreducible bounded symmetric domain $[\mathrm{BB}]$. See [BJ3] for a discussion and references pertaining to this question.

8.3 The Borel-Serre compactification [BS]. The original method in [BS] uses the geodesic action to define corners of rational parabolic subgroups, and these corners are used as a covering to define a partial compactification of $X$. On the other hand, the attachment method starts with a partial compactification $\bar{X}^{B S}$ of $X$, obtained by attaching for each parabolic $\mathbb{Q}$-subgroup, $e(\mathbf{P})=N_{P} \times$ $X_{\mathbf{P}}$ to $X$ by a procedure analogous to, but simpler than, 3.2. It is operated upon properly by $\Gamma$ and $\Gamma \backslash \bar{X}^{B S}$ is the Borel-Serre compactification of $\Gamma \backslash X$. If $\Gamma$ is neat $([\mathrm{B}], 17.1)$, it is a compact manifold with corners, of rank equal to the $\mathbb{Q}$-rank $r_{\mathbb{Q}}$ of $\mathbf{G}$, i.e. the common dimension of the maximal $\mathbb{Q}$-split $\mathbb{Q}$-tori of $\mathbf{G}$. In fact, it can be shown that $\Gamma \backslash \bar{X}^{B S}$ is a real analytic manifold with corners, and the self-gluing method of (4.3) and (4.4) also applies here to give a closed analytic manifold containing $\Gamma \backslash X$ as an open subset.

8.4. Next is a modification of $\Gamma \backslash \bar{X}^{B S}$ introduced by S. Zucker [Z] in order to study $L_{2}$-cohomology, now called the reductive Borel-Serre compactification. First a partial compactification $\bar{X}^{R B S}$ of $X$ is defined as above by attaching $X_{P}$ rather than $N_{P} \times X_{P}$ to $X$ for each parabolic $\mathbb{Q}$-subgroup. Again, $\Gamma$ acts continuously on it and $(\overline{\Gamma \backslash X})^{R B S}$ is $\Gamma \backslash \bar{X}^{R B S}$. The identity on $X$ extends to a surjective morphism of $(\overline{\Gamma \backslash X})^{B S}$ onto $(\overline{\Gamma \backslash X})^{R B S}$, the fibers of which are compact nilmanifolds $\left(\Gamma \cap N_{P}\right) \backslash N_{P}$. The compactification $(\overline{\Gamma \backslash X})^{R B S}$ maps surjectively 
onto all Satake compactifications, in particular on the maximal one. In that last case, the fibers are topological tori ([BJ3], 9.4).

\section{$\S$ 9. Compactifications of $\Gamma \backslash G$}

9.1. Let $\mathbf{P}$ be as before. We can write

$$
G=N_{P} \cdot A_{\mathbf{P}} \cdot\left(M_{\mathbf{P}} \cdot K\right)
$$

Here $\left(M_{\mathbf{P}} \cdot K\right)$ stands for the set of products $u \cdot v\left(u \in M_{\mathbf{P}}, v \in K\right)$. Of course, for a given element in $M_{\mathbf{P}} \cdot K$, the factors $u, v$ are defined up to multiplication by elements of $M_{\mathbf{P}} \cap K$, but the components of $g$ in $N_{P}$ or $A_{\mathbf{P}}$ are uniquely determined. This decomposition defines the horospherical coordinates of $g$ relative to $\mathbf{P}$ and allows one to apply the attachment method to compactify $\Gamma \backslash G$. Specifically, one can mimic the constructions underlying 8.3, 8.4 to define partial compactifications $\bar{G}^{B S}$ and $\bar{G}^{R B S}$, and their quotients by $\Gamma$.

9.2. To each parabolic $\mathbb{Q}$-subgroup $\mathbf{P}$, is associated a boundary component $e(\mathbf{P})=N_{P} \times\left(M_{\mathbf{P}} K\right)$ with the obvious definition of convergence: the sequence

$$
y_{j}=\left(n_{j}, a_{j}, z_{j}\right), \quad\left(n_{j} \in N_{P}, a_{j} \in A_{\mathbf{P}}, z_{j} \in\left(M_{\mathbf{P}} K\right)\right), \quad(j=1,2, \ldots)
$$

converges to $(n, z)\left(n \in N_{P}, z \in\left(M_{\mathbf{P}} K\right)\right.$ if $n_{j} \rightarrow n, z_{j} \rightarrow z$ and $a_{j}^{\alpha} \rightarrow \infty$ for all $\Phi\left(\mathbf{P}, A_{\mathbf{P}}\right)$. Here $\Phi\left(\mathbf{P}, A_{\mathbf{P}}\right)$ is the analogue of the $\Phi\left(P, A_{P}\right)$ in 2.3 , and is the set of weights of $A_{\mathbf{P}}$ acting on $\mathfrak{n}_{\mathbf{P}}$. Again, there are similar definitions for convergence of sequences in $e(\mathbf{P})$ to points in $e(\mathbf{Q})$ if $\mathbf{Q} \subset \mathbf{P}$. It can be shown that $\Gamma$ acts properly on $\bar{G}^{B S}$, and the quotient $\Gamma \backslash \bar{G}^{B S}$ is compact, Hausdorff. The group $K$ acts by right translation on $\bar{G}^{B S}$, commutes with $\Gamma$, hence acts also on $\Gamma \backslash \bar{G}^{B S}$, freely if $\Gamma$ is torsion-free, and the quotient is $\Gamma \backslash \bar{X}^{B S}$. If $\Gamma$ is neat, the action is free and $\bar{G}^{B S}$ is a principal $K$-bundle over $\bar{X}^{B S}$.

9.3. Similarly, the partial compactification $\bar{G}^{R B S}$ is defined by attaching for each $\mathbf{P}$ the set $e(\mathbf{P})=\left(M_{\mathbf{P}} \cdot K\right)$ as a boundary component. The convergence of sequences is defined as above, except that there is no condition on the $n_{j}$ 's. Again, $\bar{G}^{R B S}$ is Hausdorff, $\Gamma$ operates continuously on it on the left and $\Gamma \backslash \bar{G}^{R B S}$ is compact, Hausdorff. A chief difference with the previous case is that now not only $K$, but also $G$ acts on $\bar{G}^{R B S}$ and $\Gamma \backslash \bar{G}^{R B S}$ by right translation (leaving each boundary component stable). The quotient of $\Gamma \backslash \bar{G}^{R S S}$ by $K$ is $\Gamma \backslash \bar{X}^{R B S}$.

9.4. Let $H$ be any compact subgroup of $G$. Dividing out by $H$ the spaces $\Gamma \backslash \bar{G}^{B S}$ and $\Gamma \backslash \bar{G}^{R B S}$, we get compactifications of the space $\Gamma \backslash G / H$. If $X$ is a bounded symmetric domain and $G / H$ is a Griffiths period domain, the first compactification is the compactification $\Gamma \backslash D_{B S}$ in the preprint $[\mathrm{KU}]$ we have just received.

9.5. The two previous compactifications were obtained by the attachment method. Under a further assumption on $\Gamma$, a third one can be defined by the embedding method [BJ1]. We assume $\mathbf{G}$ to be simple over $\mathbb{Q}$. Then, since $\Gamma$ 
is not cocompact, no proper factor of $G$ is compact, hence any cofinite discrete subgroup is Zariski-dense [Bo1]. Moreover, by [KM], the covolumes of discrete subgroups of $G$ have a strictly positive lower bound. As a consequence, $\Gamma$ is contained as a subgroup of finite index in a maximal discrete subgroup $\Gamma^{\prime}$, and the latter is equal to its own normalizer. However, $\Gamma^{\prime}$ may not be contained in $\mathbf{G}(\mathbb{Q})$, but it is automatically so if $\mathbf{G}$ is of adjoint type. Let us now assume that $\Gamma$ is maximal discrete, and therefore equal to its normalizer. Let $\mathcal{S}(G)$ be the space of closed subgroups of $G,\left([\mathrm{Br}]\right.$, VIII, $\left.\S 5, \mathrm{n}^{\circ} 3\right)$. It is a compact Hausdorff $G$-space, $G$ acting on it by conjugation. Then the map $i_{\Gamma}$ which associates to $x \in \Gamma \backslash G$ its isotropy group in $G$ is an injective map of $\Gamma \backslash G$ into $\mathcal{S}(G)$. Using the fact that $\Gamma$ is arithmetic, $i_{\Gamma}$ can be shown to be an embedding. The closure of $i_{\Gamma}(\Gamma \backslash G)$ in $\mathcal{S}(G)$ is then a compactification of $\Gamma \backslash G$, to be denoted $(\overline{\Gamma \backslash G})^{s b}$.

9.6. Proposition. We keep the previous notation and the maximality assumption on $\Gamma$.

(i) The closed subgroups in the complement of $i_{\Gamma}(\Gamma \backslash G)$ in $(\overline{\Gamma \backslash G})^{\text {sb }}$ are conjugates of the subgroups $(\Gamma \cap P) \cdot N_{\mathbf{P}}(\mathbf{P}$ proper parabolic subgroup defined over $\mathbb{Q})$.

(ii) The identity map of $\Gamma \backslash G$ extends to a continuous surjective $G$-morphism of $\overline{\Gamma \backslash G}^{R B S}$ onto $\overline{\Gamma \backslash G}^{\text {sb }}$, which is an isomorphism if $(\Gamma \cap P) /\left(\Gamma \cap N_{\mathbf{P}}\right)$ is maximal discrete in $M_{\mathbf{P}}$ and $M_{\mathbf{P}}$ is semisimple for all $\mathbf{P}$ 's.

The proof makes use of reduction theory and of an argument occuring in the proof of the convergence of Eisenstein series for large values of the parameters. Indeed, the subgroups in $(i)$ are those which leave invariant the constant terms of cuspidal Eisenstein series. For details see [BJ1].

The assumption of $(i i)$ is e.g. satisfied if $\Gamma=\mathbf{S L}_{n}(\mathbb{Z}), \mathbf{S p}_{2 n}(\mathbb{Z})$, (or more generally if $\Gamma$ is an arithmetic subgroup associated to a $\mathbb{Z}$-form of a simply connected $\mathbb{Q}$-split $\mathbb{Q}$-group).

\section{$\S 10$. Acknowledgments}

The second author is partially supported by NSF grants and an Alfred P. Sloan Research Fellowship.

\section{References}

[BB] W. Baily, A. Borel, Compactification of arithmetic quotients of bounded symmetric domains, Ann. of Math. (2) 84 (1966), 442-528; A. Borel, C.P. II, 442-528.

[Bo1] A. Borel, Density properties for certain subgroups of semi-simple groups without compact factors, Ann. of Math. (2) 72 (1960), 179-188; C.P. II, 125-134.

[Bo2] , Automorphic forms on $\mathbf{S L}_{2}(\mathbb{R})$, Cambridge Tracts in Mathematics, 130. Cambridge University Press, Cambridge, 1997.

[BJ1] A. Borel, L. Ji, Compactifications of locally symmetric spaces, preprint, 2001.

[BJ2] _ Compactifications of symmetric spaces I, preprint, 2001.

[BJ3] Lectures on compactifications of symmetric and locally symmetric spaces, to appear in Proc. European School in Group Theory 2001.

[BJ4] _ Compactifications of symmetric and locally symmetric spaces, to be published in Mathematics: Theory and applications, Birkhäuser-Boston. 
[BS] A. Borel, J.P. Serre, Corners and arithmetic groups, Comment. Math. Helv. 48 (1973), 436-491; A. Borel, C.P. III, 244-292.

[Br] N. Bourbaki, Intégration, Chapitre 8: Convolution et reprsentations, Hermann, Paris, 1963.

[CGMP] C. De Concini, M. Goresky, R. MacPherson, C. Procesi, On the geometry of quadrics and their degenerations, Comment. Math. Helv. 63 (1988), 337-413.

[EL] S. Evens, J. Lu, On the variety of Lagrangian subalgebras. I, Ann. Sci. École Norm. Sup. (4) 34 (2001), 631-668.

[GJT] Y. Guivarc'h, L. Ji, J.C. Taylor, Compactifications of symmetric spaces, Progr. Math. 156, Birkhäuser, Boston, 1998.

[J1] L. Ji, Satake and Martin compactifications of symmetric spaces are topological balls, Math. Res. Lett. 4 (1997), 79-89.

[KU] K. Kato, S. Usui, Borel-Serre spaces and spaces of SL(2)-orbits, to appear in Proc. of World Symp. Algebraic Geometry 2000, Azumino, Advanced Studies in Pure Math., Japanese Math. Soc.

[KM] D. Kazhdan, G. Margulis, A proof of Selberg's hypothesis, Mat. Sb. (N.S.) 75 (117) (1968), 163-168.

[O1] T. Oshima, A realization of Riemannian symmetric space, J. Math. Soc. Japan 30 (1978), 117-132.

[O2] A realization of semisimple symmetric spaces and construction of boundary value maps, Representations of Lie groups, Kyoto, Hiroshima, 1986, 603-650, Adv. Stud. Pure Math., 14, Academic Press, Boston, MA, 1988.

[OS1] T. Oshima, J. Sekiguchi, Eigenspaces of invariant differential operators on an affine symmetric space, Invent. Math. 57 (1980), 1-81.

[OS2] - The restricted root system of a semisimple symmetric pair, Group representations and systems of differential equations (Tokyo, 1982), 433-497, Adv. Stud. Pure Math., 4, North-Holland, Amsterdam, 1984.

[Sa1] I. Satake, On representations and compactifications of symmetric spaces, Ann. of Math. (2) 71 (1960), 77-110.

[Sa2] - On compactifications of the quotient spaces for arithmetically defined discontinuous groups, Ann. of Math. (2) 72 (1960), 555-580.

[Sp1] T. A. Springer, Some results on algebraic groups with involutions, Algebraic groups and related topics (Kyoto/Nagoya, 1983), 525-543, Adv. Stud. Pure Math., 6, NorthHolland, Amsterdam, 1985.

[Sp2] - Algebraic groups with involutions, Proceedings of the 1984 Vancouver conference in algebraic geometry, 461-471, CMS Conf. Proc., 6, Amer. Math. Soc., Providence, RI, 1986.

[W] H. Whitney, Elementary structure of real algebraic varieties, Ann. of Math. (2) 66 (1957), 545-566; C.P. I, 456-467.

[Zu1] S. Zucker, $L_{2}$ cohomology of warped products and arithmetic groups, Invent. Math. 70 (1982/83), 169-218.

School of Mathematics, Institute for Advanced Study, Princeton, NJ 08540, U.S.A.

E-mail address: borel@math.ias.edu

Department of Mathematics, University of Michigan, Ann Arbor, Mi 48109, U.S.A.

E-mail address: lji@math.las.umich.edu 